\title{
SOLITARY WAVE INTERACTION WITH A SUBMERGED PERMEABLE BREAKWATER: EXPERIMENT AND NUMERICAL MODELING
}

\author{
Yun-Ta $\mathrm{Wu}^{1}$, Shih-Chun Hsiao ${ }^{2}$ and Guan-Shiue $\mathrm{Chen}^{3}$
}

\begin{abstract}
We study the interactions between a non-breaking solitary wave and a submerged permeable breakwater experimentally and numerically. The particle image velocimetry (PIV) technique was employed to measure instantaneous free surface displacements and velocity fields in the vicinity of the porous media. The porous media, consisted of uniform glass-made spheres, was mounted on the seafloor. Quantitative mean properties were obtained by ensemble averaging 30 repeated instantaneous measurements. In addition, two different numerical considerations are taken to simulate the experiments. One is to model an idealized volume-averaged porous media using a twodimensional (2D) volume of fluid (VOF)-type model. This model is based on the Volume-Averaged ReynoldsAveraged Navier-Stokes (VARANS) equations coupled with the non-linear $k-\varepsilon$ turbulence closure solver. The other is to model the real porous breakwater constructed by spheres using a three-dimensional (3D) VOF-type model. This model solves 3D incompressible Navier-Stokes equations with Large-eddy-simulation (LES) model. The comparisons were performed between measurements, 2D and 3D numerical results for the time histories of the free surface elevation, instantaneous free surface displacements and corresponding velocity properties around the permeable object. Fairly good agreements were obtained. The verified 3D numerical results were used to trace the trajectories of fluid particle around the porous media to help understand the possible sediment movements in suspended loads. Also, the 2D numerical model is used to estimate the energy reflection, transmission and dissipation using the energy integral method by varying the aspect ratio and the grain size of the permeable obstacle.
\end{abstract}

Keywords: solitary wave; submerged permeable breakwater; PIV; RANS model; LES model

\section{INTRODUCTION}

Submerged permeable breakwaters have been frequently built along the coast to reduce the impacts from waves and currents not only due to their lower costs than impermeable structures but also because they can dissipate more wave energy through viscous dissipation within the porous media. As a result, water wave interaction with submerged permeable structures has received a large amount of attentions, especially under the attacks by nonlinear long waves. In many related literatures, solitary waves have been considered to model certain behavior of nonlinear long waves, such as leading wave of tsunamis and storm surges (Synolakis and Bernard, 2006). For numerical simulation point of view, a solitary wave that has only single wave crest can be used to study the wave transformation and associated turbulent velocity fields separately without the effects by both the preceding and subsequent waves.

It is well known that the functional efficiency of porous breakwater is assessed in terms of the wave reflection, transmission, and dissipation (RTD) coefficients in usual practice. Many studies provide detailed estimation of RTD coefficients for emerged-type porous obstacle under a solitary wave (Lin and Karunarathna, 2007; Lynett et al., 2000). The dynamical interaction between the structure itself and the induced flow fields is indispensable in the design of coastal structure. For solitary wave interaction with submerged permeable breakwater, Huang et al. (2003) studied the flow fields outside and inside an object using a two-dimensional (2D) unsteady Navier-Stokes type model and assuming the flow outside the porous media to be laminar. Lately, Lara et al. (2011) investigated the wave damping on solitary wave over an underwater porous shelf using a 2D Volume-Averaged Reynolds-Averaged Navier-Stokes (VARANS) model. However, the model validation in most existing numerical studies is carried out for the comparisons with free surface motion induced by periodic waves over a porous media. To the knowledge of the authors, detailed measurement on the interaction between a solitary wave and a submerged permeable breakwater is still lacking in literature.

In this paper, we present the comprehensive measurements of solitary wave propagation over a submerged permeable breakwater using a particle image velocimetry (PIV) system. Present laboratory experiments provide measured data for the mean time histories of the free surface elevation recorded by wave gauges and instantaneous mean free surface motion as well as mean velocity properties

\footnotetext{
${ }^{1}$ Department of Hydraulic and Ocean Engineering, National Cheng Kung University, No. 1, University Road, Tainan, 701, Taiwan.

${ }^{2}$ Department of Hydraulic and Ocean Engineering, National Cheng Kung University, No. 1, University Road, Tainan, 701 , Taiwan.

${ }^{3}$ Department of Hydraulic and Ocean Engineering, National Cheng Kung University, No. 1, University Road, Tainan, 701, Taiwan.
} 
around the porous media imaged by PIV. We use two different concepts of numerical model to simulate identical experimental conditions. One is to model an idealized volume-averaged porous media using two-dimensional (2D) VARANS model (Hsu et al., 2002; Lin and Liu, 1998), and the other one is to simulate the real permeable structure as we used in the experiment using threedimensional (3D) Large-eddy-simulation (LES) model (Hu et al., 2012; Wu, 2004) to study 3D effects. The comparisons between experiments, 2D and 3D results were carried out for the time histories of the free surface elevation, instantaneous free surface motion and corresponding velocity properties as a mean of numerical model validation. Then, we use the validated 3D numerical results to trace the trajectories of fluid particle around the permeable breakwater, and the 2D numerical model is employed as a tool to further explore the functional efficiency of the submerged porous media under solitary wave forcing via varying the porosity and the length of the breakwater.

\section{EXPERIMENT}

New experiments were conducted in a two-dimensional glass-walled and glass-bottomed wave flume at Department of Hydraulic and Ocean Engineering, National Cheng Kung University. The physical wave tank is $25 \mathrm{~m}$ long, $0.5 \mathrm{~m}$ wide and $0.6 \mathrm{~m}$ deep. Figure 1 schematically depicts the experimental facilities and apparatus layouts. Solitary waves were generated by a digital servocontrolled piston-type wavemaker at one end of the wave flume using the procedure suggested by Goring (1978). The target solitary wave with a wave height $(H)$ of $4.77 \mathrm{~cm}$ in a constant water depth $(h)$ of $10.6 \mathrm{~cm}$ (i.e. $H / h=0.45$ ) was given throughout in present experiment. An idealized permeable breakwater (i.e. aspect ratio a / b $=2.0$ ) was mounted on the seafloor with a constant value of $13 \mathrm{~cm}$ in width (a) and $6.5 \mathrm{~cm}$ in length (b), consisted of uniform glass-made spheres with a constant diameter of $1.50 \mathrm{~cm}$ to yield a porosity value of 0.52 . The origin coordinate system $(x, y)=(0,0)$ was defined at the intersection of the bottom and the left side of the breakwater.

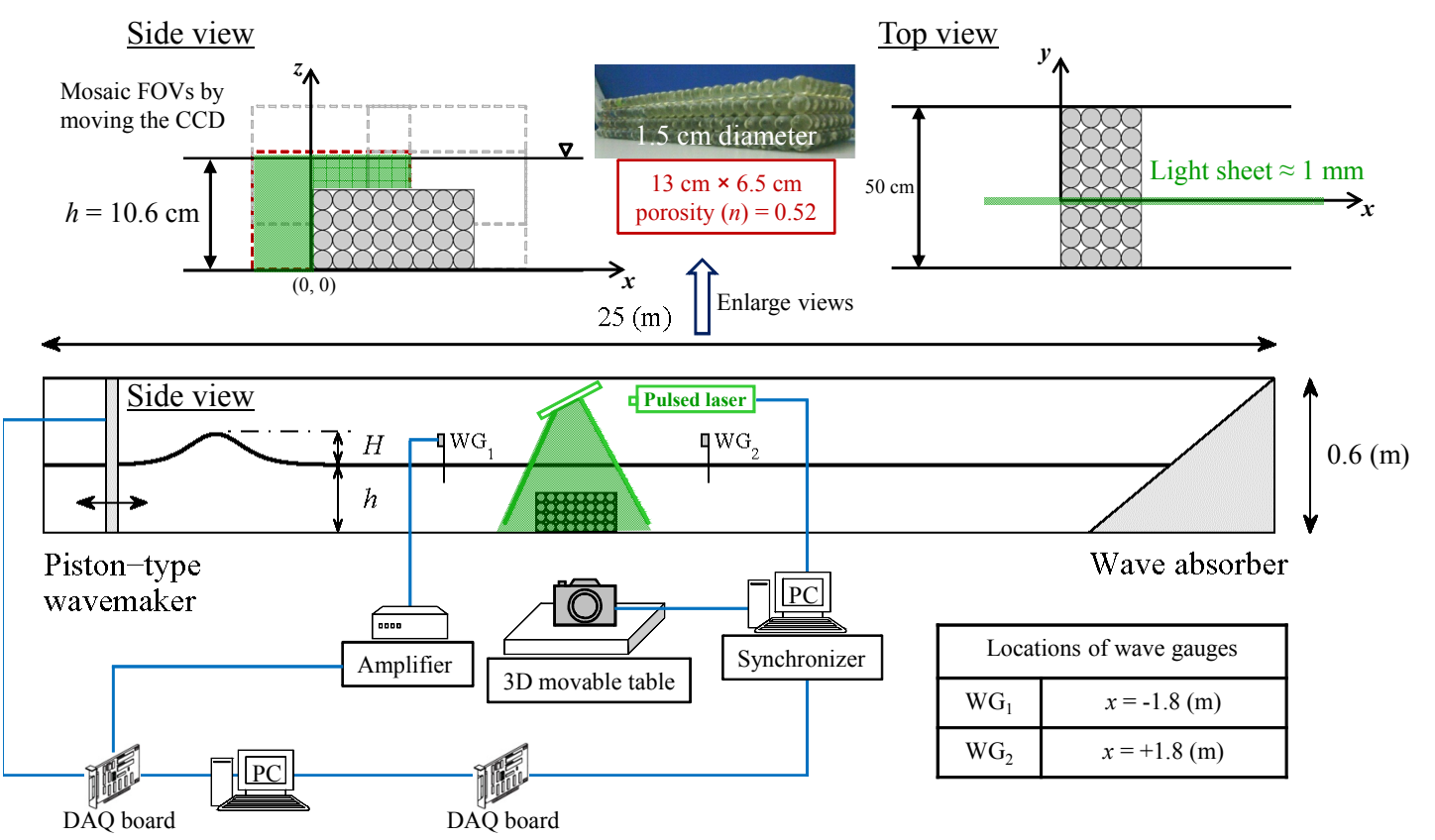

Figure 1. Overview of experimental set-up and facilities (not to scale).

Velocity fields in the vicinity of the permeable breakwater were measured by a PIV system. A 12bit digital CCD camera with a resolution of $1600 \times 1200$ pixels was used to capture instantaneous particle images. The framing rate is 20 frames per second (fps) to yield a temporal resolution of 10 phases per second. Four PIV fields of view (FOVs) were measured in order to form a continuous flow fields around the porous media. The size of each FOV was $186.04 \times 139.53 \mathrm{~mm}^{2}$. The light source to illuminate the measuring section was a dual-head Nd:YAG pulsed laser system with a maximum energy output of $120 \mathrm{~mJ}$ per pulse in $532 \mathrm{~nm}$ wavelength and a repetition rate of $20 \mathrm{~Hz}$. To avoid the wall effect, the vertical light sheet with a thickness about $1 \mathrm{~mm}$ was formed a distance away from the 
side wall. The water in the wave tank was seeded by tiny buoyant hollow spherical glass particles with a mean diameter of $10 \mu \mathrm{m}$ and a specific gravity of $1.05 \mathrm{~g} / \mathrm{cm}^{3}$. The velocity field was calculated via a multi-pass algorithm with an interrogation window size of $24 \times 24$ pixels and a $50 \%$ overlap. Based on the resolution of PIV camera, the size of FOV and interrogation window, the spatial resolution of current PIV system is about a value of $1.4 \mathrm{~mm}$. Velocities were determined by the cross-correlation technique using FFT and three-point Gaussian peak-finding algorithms (Raffel et al., 1998). Certain criteria were set to retain the calculated velocity vectors (Adrian, 1991), while outliers were removed using a dynamic mean value filter and a local median filter (Raffel et al., 1998).

The time history of the surface wave profiles were measured by two capacitance-type wave gauges in front of $(x=-1.8 \mathrm{~m})$ and behind $(x=+1.8 \mathrm{~m})$ the breakwater. The first one was chose as the reference gauge, which means as the wave crest arrives at the first wave gauge is defined as $t=0 \mathrm{~s}$. In addition, a total of 30 runs for each FOV were conducted under identical experimental conditions for computing ensemble-averaging quantities.

\section{NUMERICAL MODEL}

\section{D VARANS Model}

The computational model, named COBRAS (COrnell BReaking And Structure), is a twodimensional numerical scheme which solves the Reynolds-Averaged Navier-Stokes (RANS) equations (flow outside the porous media) and the Volume-Averaged Reynolds Averaged Navier-Stokes (VARANS) equations (flow inside the porous media) for describing the mean (ensemble averaged) flow field with the nonlinear $k-\varepsilon$ equations for the turbulent kinetic energy (TKE), $k$, and the turbulent dissipation rate, $\varepsilon$. The model solved the RANS and VARANS equations by using the finite-difference two-step projection method (Chorin, 1968). Also, the volume of fluid (VOF) method was constructed for tracing the free surface motion originally proposed by Hirt and Nichols (1981). More detailed information about the numerical implementation can be found in studies of Lin and Liu (1998) and Hsu et al. (2002).

The boundary conditions for the mean flow field were the no-slip condition at the solid boundaries and the zero-stress condition applied to the mean free surface for neglecting the air-flow effect, respectively. For the turbulence field, a log-law distribution of the mean tangential velocity within the turbulent boundary layer was taken into account near the solid boundary. Additionally, the surface tension effect was not considered. In the downstream boundary, the radiation condition was utilized for allowing the wave outgoing the computational domain without significant reflection.

In the present model, a desired solitary wave was generated by sending the free surface elevation and the velocity components through upstream boundary using conventional Boussinesq theory derived by Lee et al. (1982). Additionally, the active absorption inflow boundary condition was utilized in inflow boundary to avoid re-reflection (Torres-Freyermuth et al., 2010).

\section{D LES Model}

The present 3D numerical model, Truchas, solves the 3D incompressible Navier-Stokes equations with the Large-Eddy-Simulation module for multiphase transient fluid flow. The VOF algorithm is adopted to trace the free surface motion, and the parallel computational technique is used to speed up the simulations by using MPICH on Linux system. Detailed information on the numerical implementation can be found in the studies of $\mathrm{Wu}$ (2004) and $\mathrm{Hu}$ et al. (2012).

\section{Computational Domain}

For 2D numerical simulation, a numerical wave flume consists of the length of $6.0 \mathrm{~m}$ with the height of $0.2 \mathrm{~m}$. Two numerical wave gauges is employed at the same relative location as in experiment in order to check the wave forms.

The 3D LES modeling is used to simulate identical porous structure composed by a series of uniform sphere as we used in experiments. The 3D porous structure is constructed by the partial-cell method. The numerical wave tank in $x-z$ direction is identical to $2 \mathrm{D}$ modeling. To simplify the simulation in $y$ direction, we only use one full sphere surrounded by two half-spheres and use the freeslip boundary condition at the lateral boundaries to mirror three full spheres. This simulation runs on a PC cluster with 32 processors that still need at least 8 days. In the lateral direction, the measurement section slices on the quarter of a sphere to avoid the laser beam reflection from the glass-made sphere. Here we only present the numerical results in the same section as in PIV measurement to compare with experimental data. 


\section{RESULTS AND COMPARISONS}

\section{Comparisons between Measured and Modeled Results}

To model a volume-averaged porous media, three empirical coefficients need to be specified in the numerical model, i.e., linear friction $\alpha$, non-linear friction $\beta$ and added mass coefficient $\gamma$. Some studies attempt to determine those coefficients by conducting physical experiment, such as van Gent (1995) and Liu et al. (1999). Recently, Lara et al. (2011) proposes a set of mathematical formula to determine those coefficients. Detailed comparisons of those different values of empirical coefficients can be found in Table 1. Since those coefficients have been widely used in the porous media flow modeling, we would like to use those different values into the numerical model to examine their difference and also compare with our experiments.

\begin{tabular}{|c|c|c|c|}
\hline \multicolumn{4}{|c|}{ Table 1. Comparisons of various empirical coefficients. } \\
\hline Authors & $\alpha$ & $\beta$ & $\gamma$ \\
\hline van Gent (1995) & 1000.00 & 1.10 & 0.34 \\
Liu et al. (1999) & 200.00 & 1.10 & 0.34 \\
Lara et al. (2011) & 724.57 & 8.15 & 0.34 \\
\hline
\end{tabular}

The first comparison is given for the time histories of free surface elevation. As can be seen in Fig. 2, three numerical results using 2D VARANS modeling fit the measurements fairly well for the incident wave, reflected waves and transmitted waves. Little overestimation of reflected waves comes from the numerical results using the empirical coefficients suggested by Lara et al. (2011). Not surprisingly, we also find that the higher the reflected wave is, the more impermeable the porous structure becomes. Among those empirical coefficients we used, the calculated result using the value suggested by Liu et al. (1999) is relatively appropriate and accurate to compare with our experiments. In the following comparisons with 3D numerical results, only the results of $2 \mathrm{D}$ VARANS modeling were used as a representative.

The comparisons of the time histories of free surface elevation again show reasonably good agreements between experiment, 2D and 3D numerical modeling for incident wave, reflected and transmitted waves (Fig. 3). Obviously, the time histories of the free surface elevation do not have significant variation in different lateral sections for 3D numerical modeling (Fig. 3).

Since the initial forcing of solitary wave has been checked, the next comparisons would be given for the instantaneous free surface elevation and corresponding velocity profiles in both horizontal and vertical directions. For PIV measurements, the velocity within the porous media cannot be measured accurately due to the lack of optical access and misleading laser reflections from the glass-made spheres. Although velocity calculations may provide some results within the porous media, we do not discuss about it in this paper due to the aforementioned uncertainty. The free surfaces estimated from PIV images were detected automatically using image processing method instead of manpower.

The overall comparisons between measured, 2D and 3D modeled results are fairly well (Fig. 4). Slight difference exists for the velocity characteristics calculated by 2D VARANS model as the leading wave front approaches the breakwater (Fig. 4 (a): $x=0.04 \mathrm{~m}$ and $x=0.08 \mathrm{~m}$ ). This is because the physically distinct porous media were considered between experiment and 2D numerical modeling. Significant variation can be found in Fig. 4 (a) at $x=0.04 \mathrm{~m}$. The 3D modeled result has a positive value for vertical velocity near the weather boundary of porous media whereas the measurement and $2 \mathrm{D}$ results show almost uniform value at that region. The reason caused this variation is because the $3 \mathrm{D}$ calculated results slice is taken on a quart of sphere that allows a velocity to be existent in the porous media. Although the PIV measurements were also sliced on the same profile, this event is invisible due to the lack of optical light accesses. In Fig. 4 (b), significant difference is visible between measured and 2D modeled results for the vertical velocity profile at $x=0.16 \mathrm{~m}$. It is well known that the vortices would be generated due to flow separation as water waves pass over an obstacle. The same scenario absolutely happens in current study. Although we do not present the spatial velocity fields in this paper, a main clockwise vortex was induced in the lee side of the porous media which is visible in both physical experiments and numerical modeling. The velocity profile at $x=0.16 \mathrm{~m}$ is just crossing the main vortex, and the physically distinct of the porous media causes the difference in the initial stage of the formation of the main vortex. On the other hands, the use of $3 \mathrm{D}$ numerical model to construct the same 3D porous breakwater can observe a similar phenomenon like experiments, and the 
calculated results fit the experiments better than 2D modeling for the initial stage of the formation of the main vortex (Fig. 4 (b)). After the wave crest passes over the breakwater, no significant variation exists between 2D and 3D numerical results (Fig. 4 (c)).
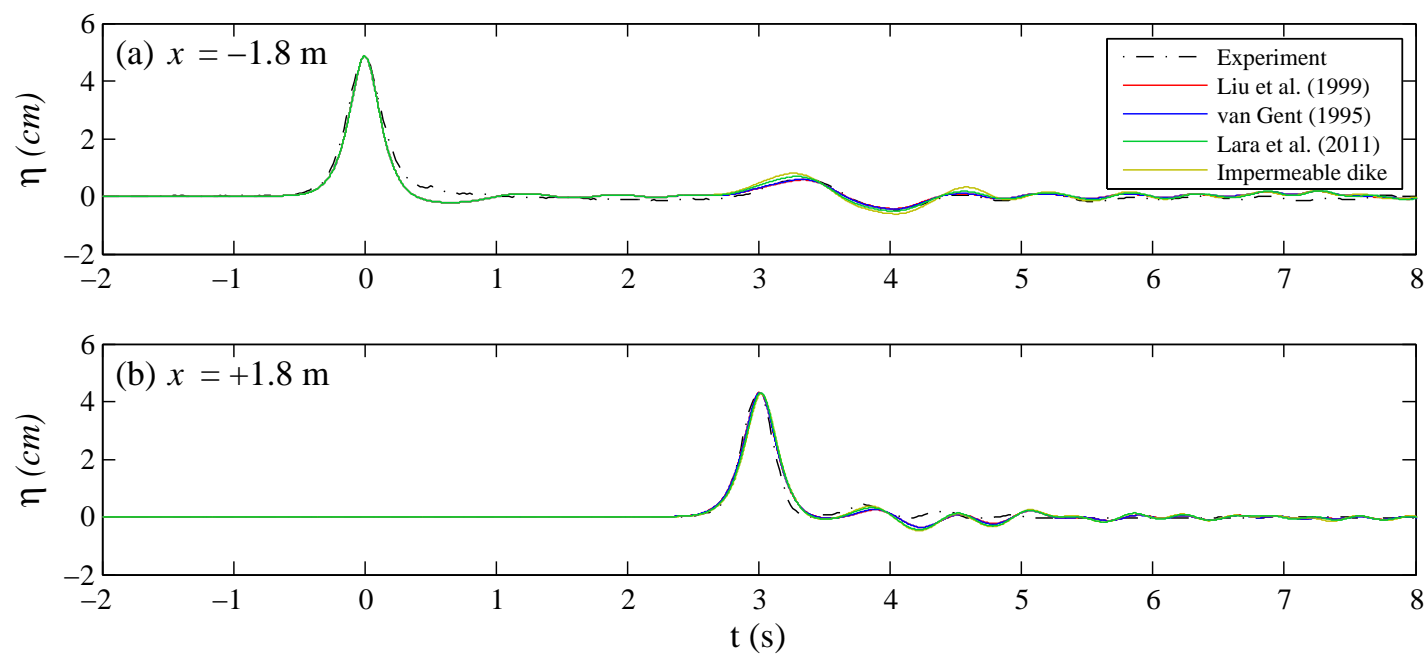

Figure 2. Comparisons of the time histories of the free surface elevation between experiments and 2D numerical results with three various empirical coefficients at (a) $x=-1.8 \mathrm{~m}$; (b) $x=1.8 \mathrm{~m}$.
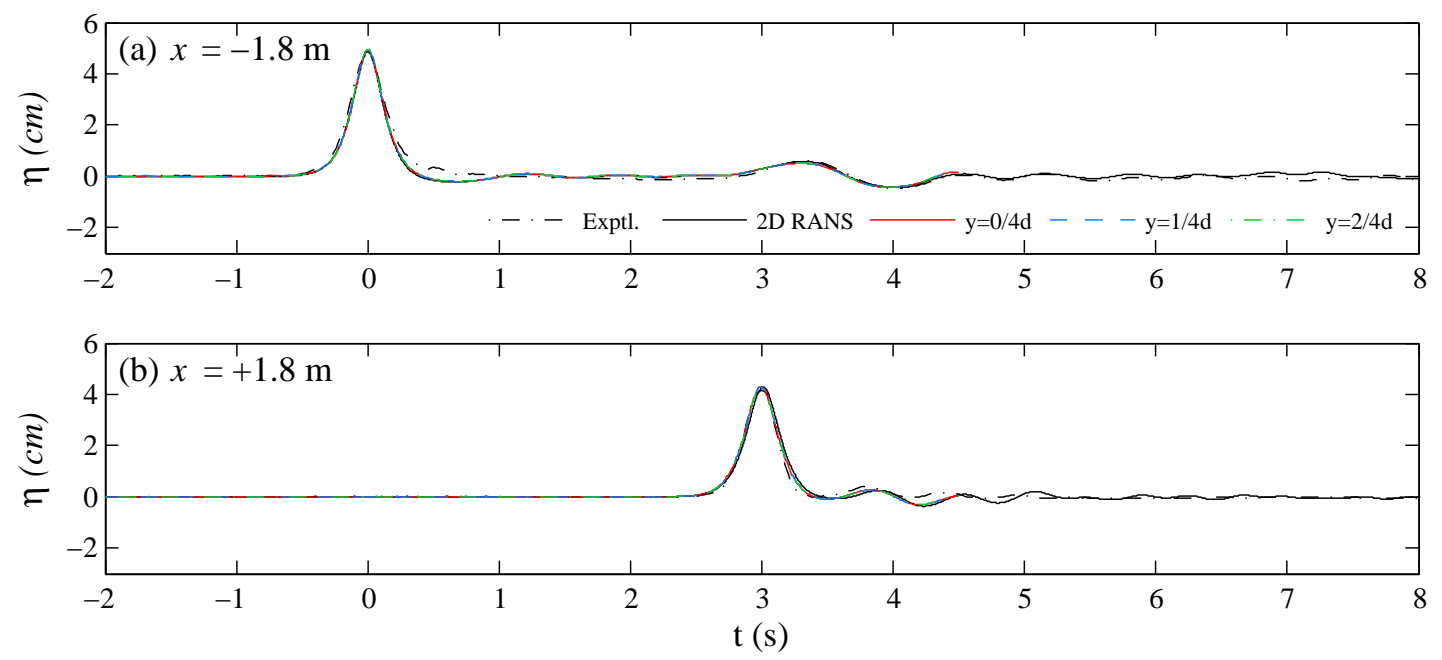

Figure 3. Comparisons of the time histories of the free surface elevation between experiments, 2D and 3D numerical results in three various lateral directions at (a) $x=-1.8 \mathrm{~m}$; (b) $x=1.8 \mathrm{~m}$. 
(a) $\mathrm{t}=1.45(\mathrm{~s})$

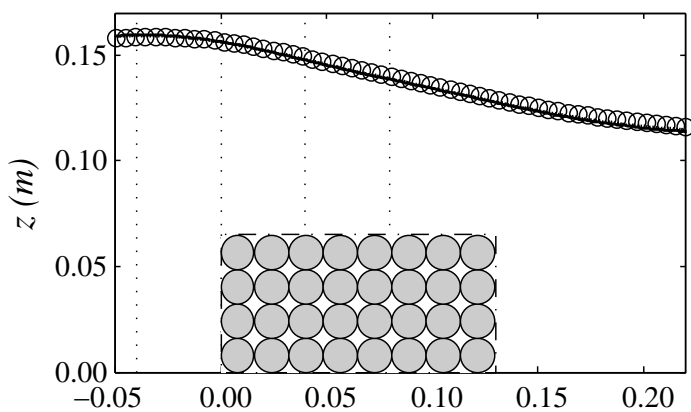

(b) $\mathrm{t}=1.65$ (s)

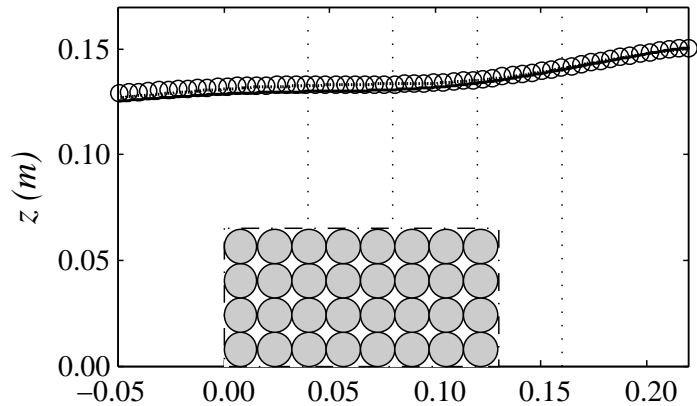

(c) $\mathrm{t}=2.05(\mathrm{~s})$

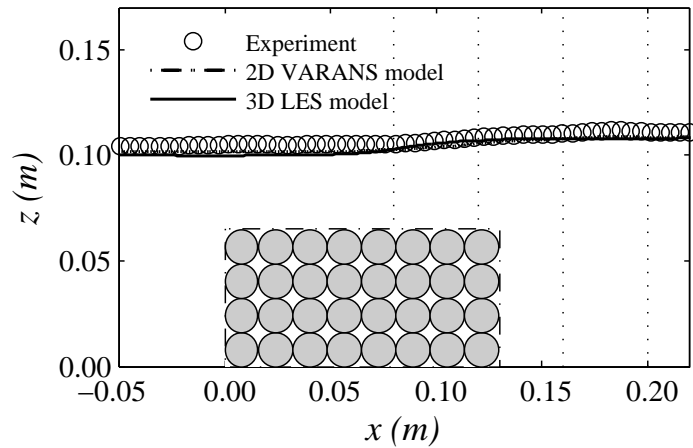

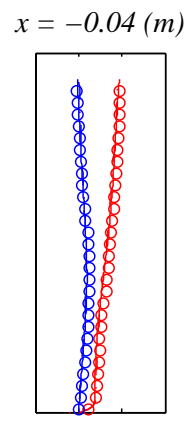

0.00 .5

$x=0.04(\mathrm{~m})$

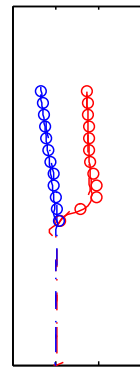

0.00 .5

$x=0.08(\mathrm{~m})$

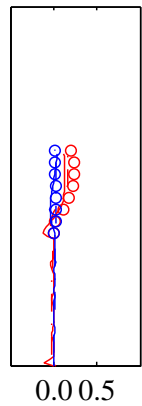

0.00 .5

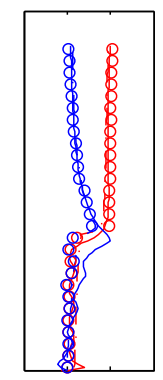

0.00 .5

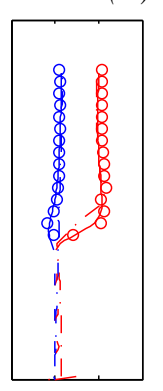

0.00 .5

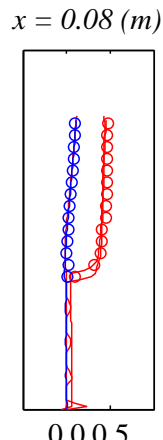

0.00 .5
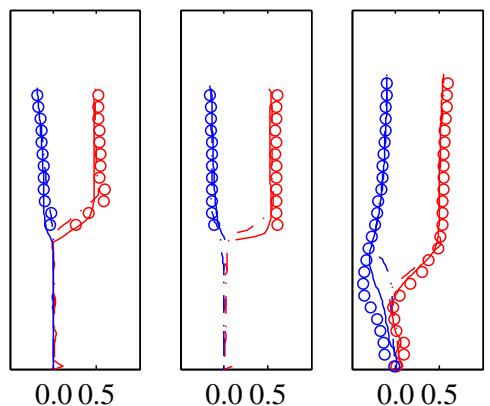

0.00 .5
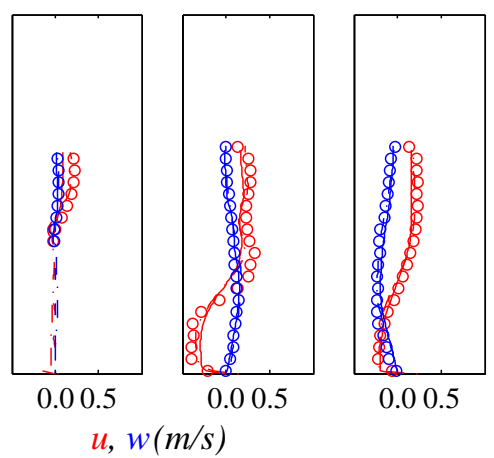

Figure 4. Comparisons between experiments and numerical results for the instantaneous free surface elevation (left) and corresponding velocity profiles (right) at three various time instants. 


\section{Trajectories of Fluid Particle around the Porous Breakwater}

From the viewpoint of engineering practices, the induced vortices and corresponding turbulence mainly contribute to sediment transport then result in scouring near the toe of coastal breakwaters. To provide more information on the possible transportation of sediment, the fluid particles with initial locations close to the breakwater were determined in order to trace their trajectories of each particle. Although the motions of fluid particles are physically distinct from that of the real sediment, it still might provide an insight into the possible sediment movement in suspension near the breakwater.

In this study, the trajectories of fluid particles is not really based on Lagrange coordinate system, is determined from the velocity of each particles in different time instant then to calculate their displacement. Therefore, we must validate this method before we use it. Recently, Hsu et al., (2012) performed measured particle trajectories for a solitary wave propagating in a constant water depth. Figure 5 shows the comparisons between experiments and numerical results using $3 \mathrm{D}$ model for three different initial locations in vertical direction of marked particle, and the agreements between measurements and calculations are fairly well. This implies using this method to determine the trajectories of fluid particle is reasonable and reliable.

Figures 6 to 8 respectively shows the calculated results of trajectories of fluid particle with initial locations close to the weather side, lee side and top of the permeable breakwater. Those particles are released at the same time from $\mathrm{t}=0.0$ to $4.0 \mathrm{~s}$ with an identical time interval of $0.01 \mathrm{~s}$. The symbols "circle" and "triangle" in Figs. 6 8 depict the initial and ending position of each marked fluid particle, respectively.

As can be seen in Fig. 6, for those particles that initially located near the weather edge of the breakwater, most of them starts offshore and moves in the onshore direction, which implies possible scouring at the foot of the coastal structure. As some of those particles are transported downstream, it would be driven by the main vortex. The fluid particles near the gap spacing may get stuck within the porous structure. Once those particles move into the breakwater, it would not penetrate the breakwater and may finally accumulate within the porous media. If those particles are considered as sand or sediment, this phenomenon may change the designed porosity to further effect the functional efficiency of the coastal breakwater.

For those particles initially located near the lee side of the breakwater (see Fig. 7), most of them would still stay in the lee side and also be driven by the main vortex. Some particles near the seafloor may finally accumulate near the junction between the seafloor and the lee side of the obstacle and their displacements were smaller than those of the other particles. Interestingly, some particles located near a quarter of sphere move in lateral direction but finally still accumulate in the lee side of obstacle.

For those particle initially located near the top of the breakwater (see Fig. 8), all of them are transported downstream by wave propagation and the induced main vortex. Most of those particles finally accumulate near the junction between the seafloor and the lee side of the obstacle. Some particle may move in the opposite direction of the wave and eventually stay within the porous breakwater.

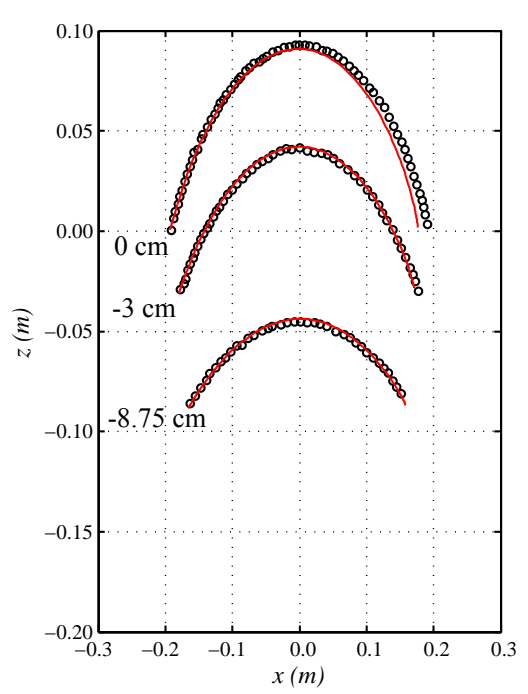

Figure 5. Comparisons between measurement (o) and 3D numerical calculations (-) in three various initial locations. 


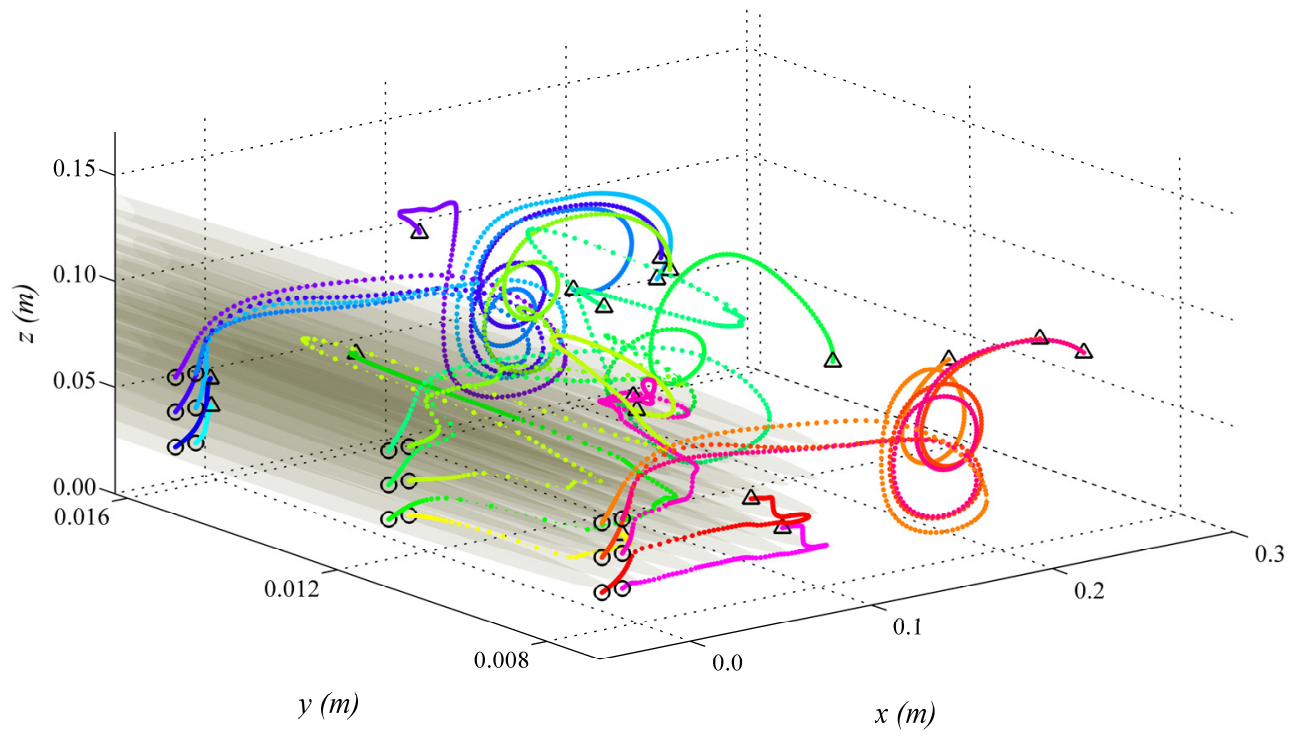

$y=0 / 4 d$
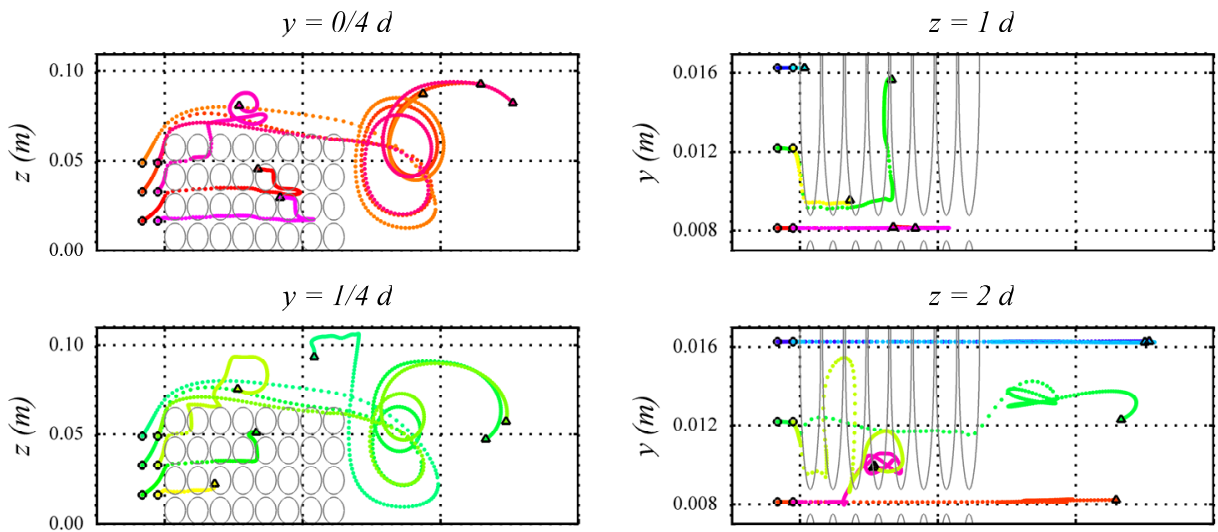

$y=2 / 4 d$

$z=3 d$
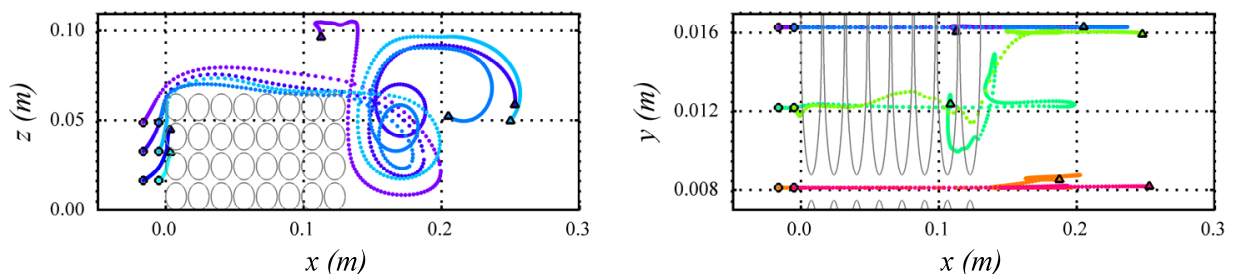

Figure 6. Simulated particle trajectories initially located near the weather side of the permeable breakwater. The top panel provides the results in 3D view, the second panel in the left side shows the results in $x-z$ plane with three lateral sections and the second panel in the right side shows the results in $x-y$ plane with three vertical sections. 

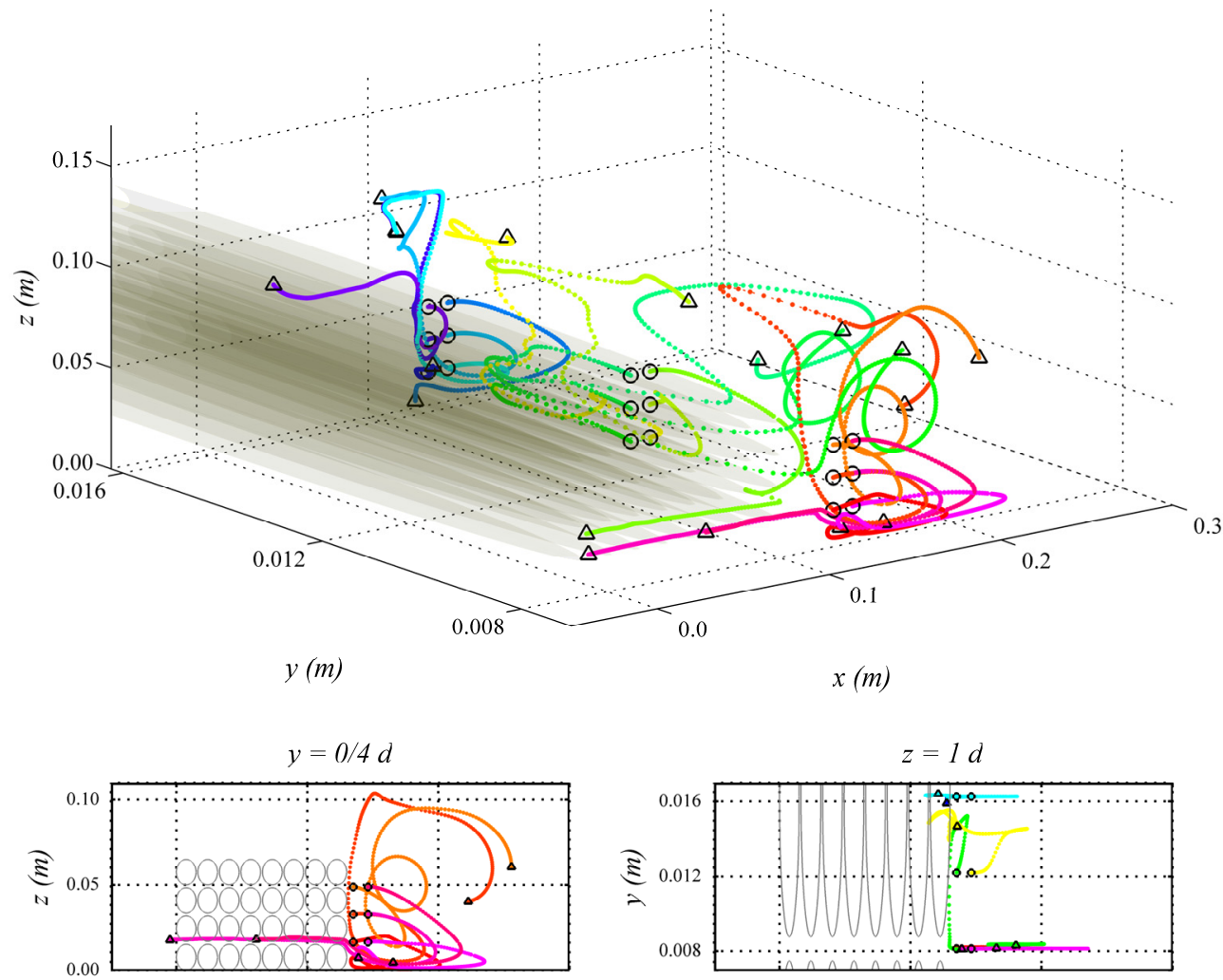

$y=1 / 4 d$

$z=2 d$
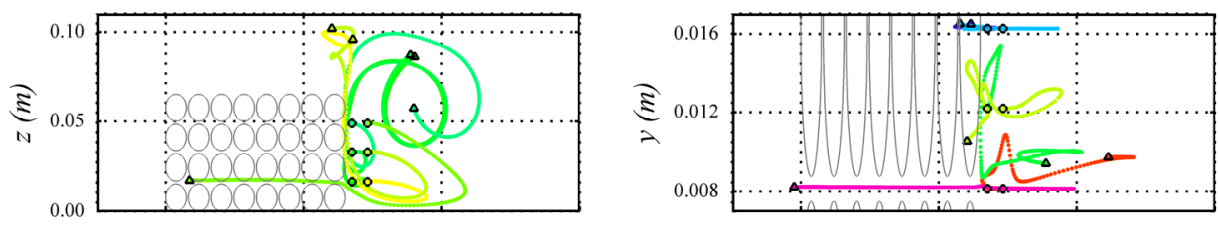

$y=2 / 4 d$

$z=3 d$
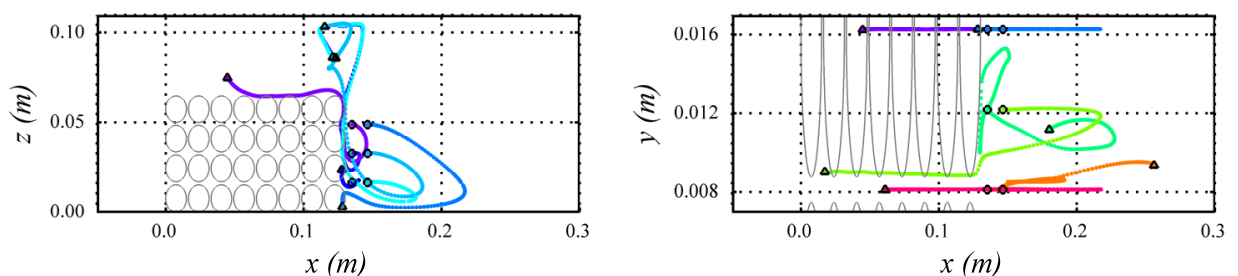

Figure 7. Simulated particle trajectories initially located near the lee side of the permeable breakwater. The top panel provides the results in 3D view, the second panel in the left side shows the results in $x-z$ plane with three lateral sections and the second panel in the right side shows the results in $x-y$ plane with three vertical sections. 

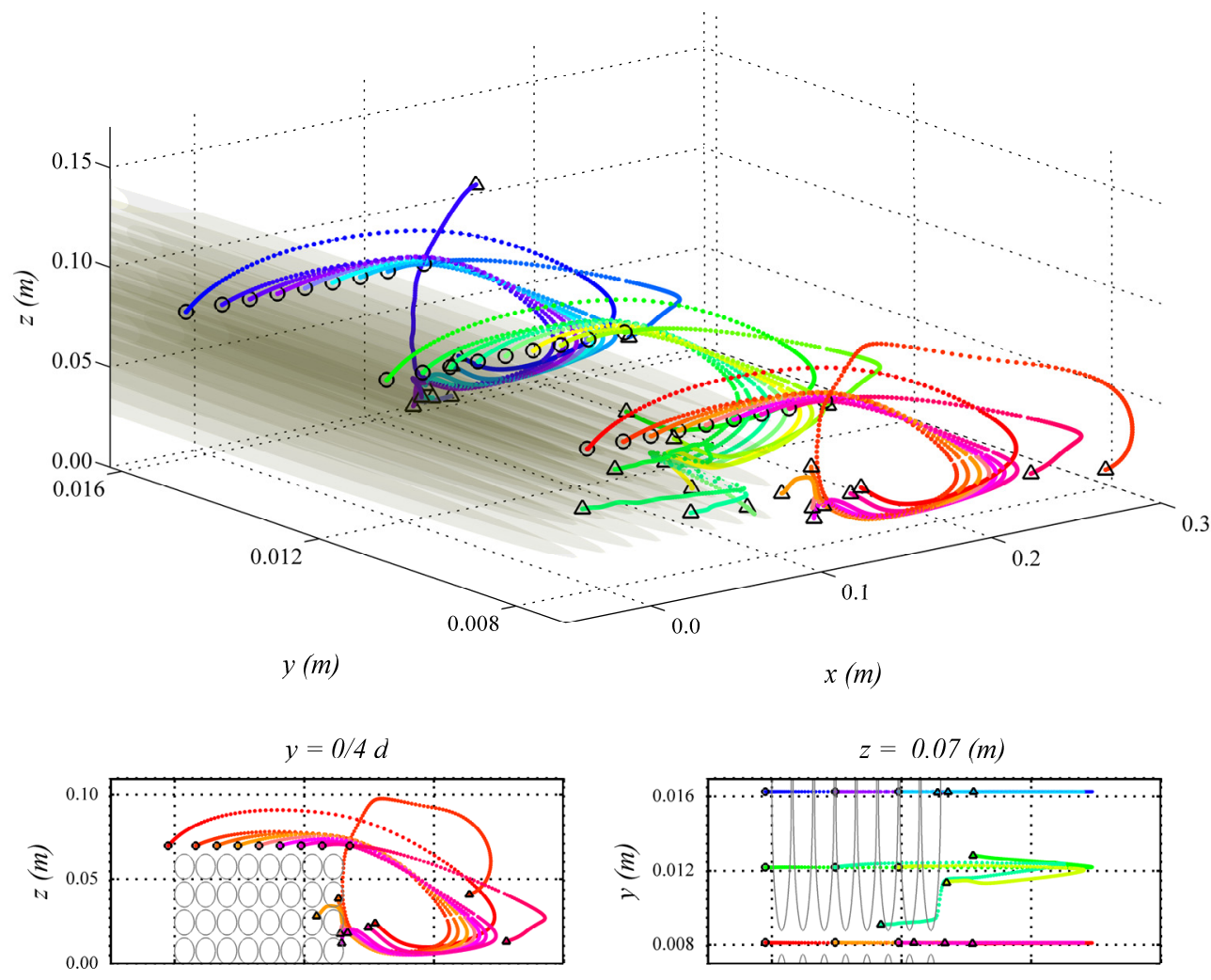

$y=1 / 4 d$
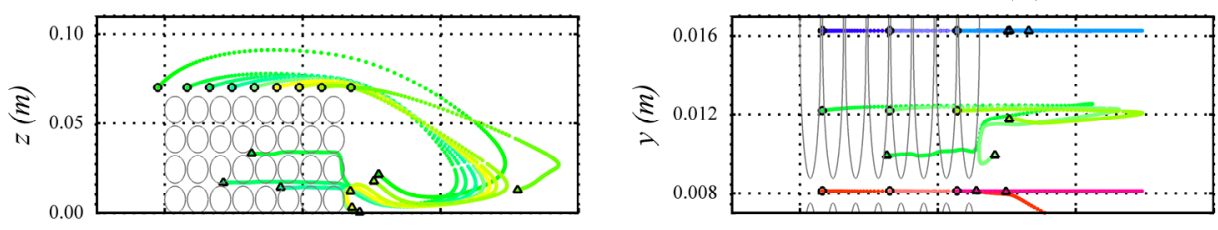

$y=2 / 4 d$
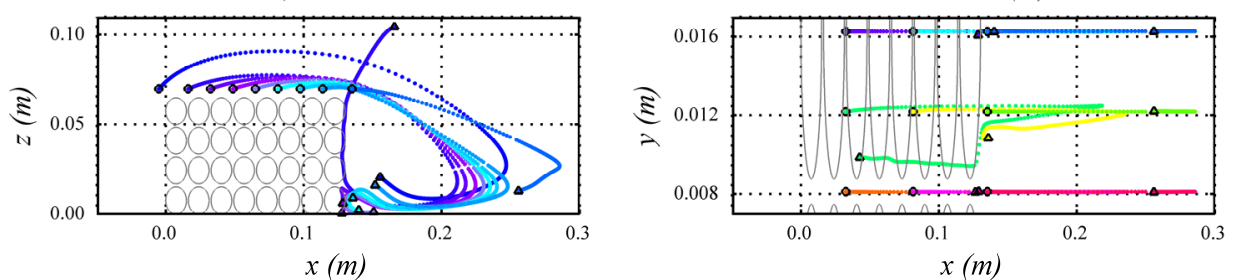

Figure 8. Simulated particle trajectories initially located near the top of the permeable breakwater. The top panel provides the results in 3D view, the second panel in the left side shows the results in $x-z$ plane with three lateral sections and the second panel in the right side shows the results in $x-y$ plane with three vertical sections. 


\section{RTD Coefficients}

We evaluate the functional efficiency of the submerged porous breakwater through the calculations of RTD coefficients using the energy integral method proposed by Lin (2004), based on integration of energy flux, instead of using wave height information only. Using this method to determine RTD coefficients is more appropriate during the wave-structure interaction under the process of vortex shedding and wave breaking. This method has been frequently used in literatures to estimate the functional efficiency of coastal structures under a solitary wave (Lin, 2004; Lin and Karunarathna, 2007; Wu et al., 2012).

To further check that the hydraulic performance of submerged permeable breakwater between two numerical predictions, the RTD coefficients obtained from $2 \mathrm{D}$ and $3 \mathrm{D}$ numerical simulations are plotted against each other (Fig. 9). The agreement is fairly well. Therefore, we can simply use the 2D VARANS numerical model as a tool to further explore the RTD coefficients with a wider range of parameters due to its calculation efficiency.

The influence of different grain size $\left(\mathrm{d}_{50}\right)$ of the submerged permeable breakwater on RTD coefficients is studied for fixed parameters of porosity and obstacle geometry. As can be seen in Fig. 10 , no significant variation on the energy transmission coefficient was found by changing particle size. For the use of relatively small particle size, the energy reflection coefficients slightly decrease and the energy dissipation coefficients slightly increase with increasing the particle size. Apart from this, the effect of particle size is minor and kept nearly constant value.

We also investigate the effect of porosity and the length of breakwater on RTD coefficients for fixed particle size. As can be seen in Fig. 11, the energy reflection coefficients decrease with increasing the porosity but the increase of the length of breakwater does not have significant effect on the energy reflection coefficients. The energy transmission coefficients remain nearly constant with increasing the porosity value from 0.0 to 0.5 . With increasing the length of the breakwater, the energy transmission coefficients decrease because more energy is damped within the permeable breakwater. The energy dissipation coefficients remain nearly constant as the porosity value changes from 0.0 to 0.8 . The influence of the length of the submerged permeable breakwater on RTD coefficients is clearly more significant. The energy reduction is found to increase with increasing the aspect ratio of obstacle due to the more energy is dissipated through the viscous friction within the lengthening porous media.

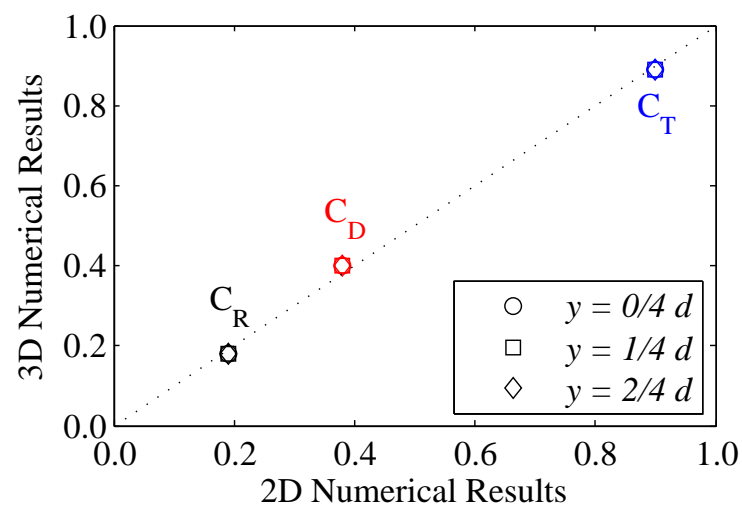

Figure 9. Direct comparisons of RTD coefficients between 2D and 3D numerical results. 


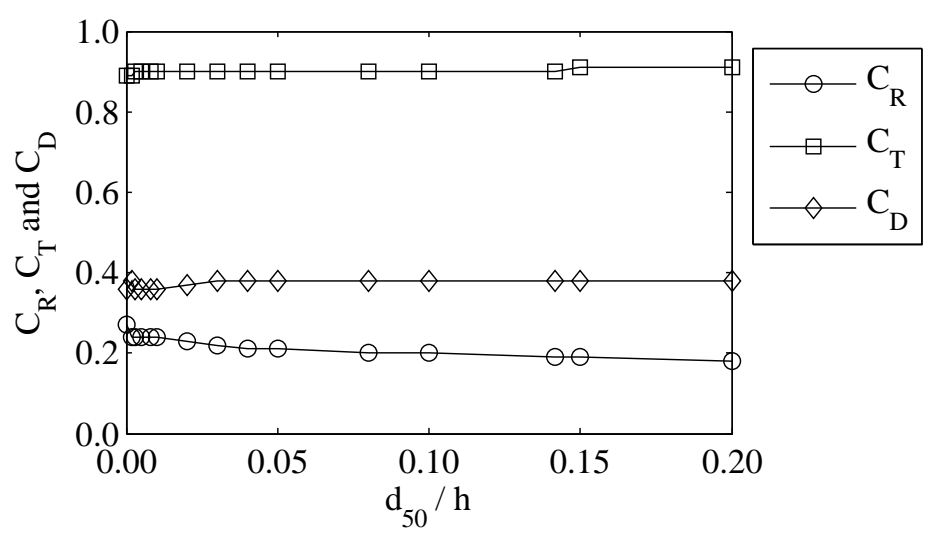

Figure 10. Effects of different particle sizes $\left(d_{50}\right)$ of the porous obstacle on RTD coefficients.
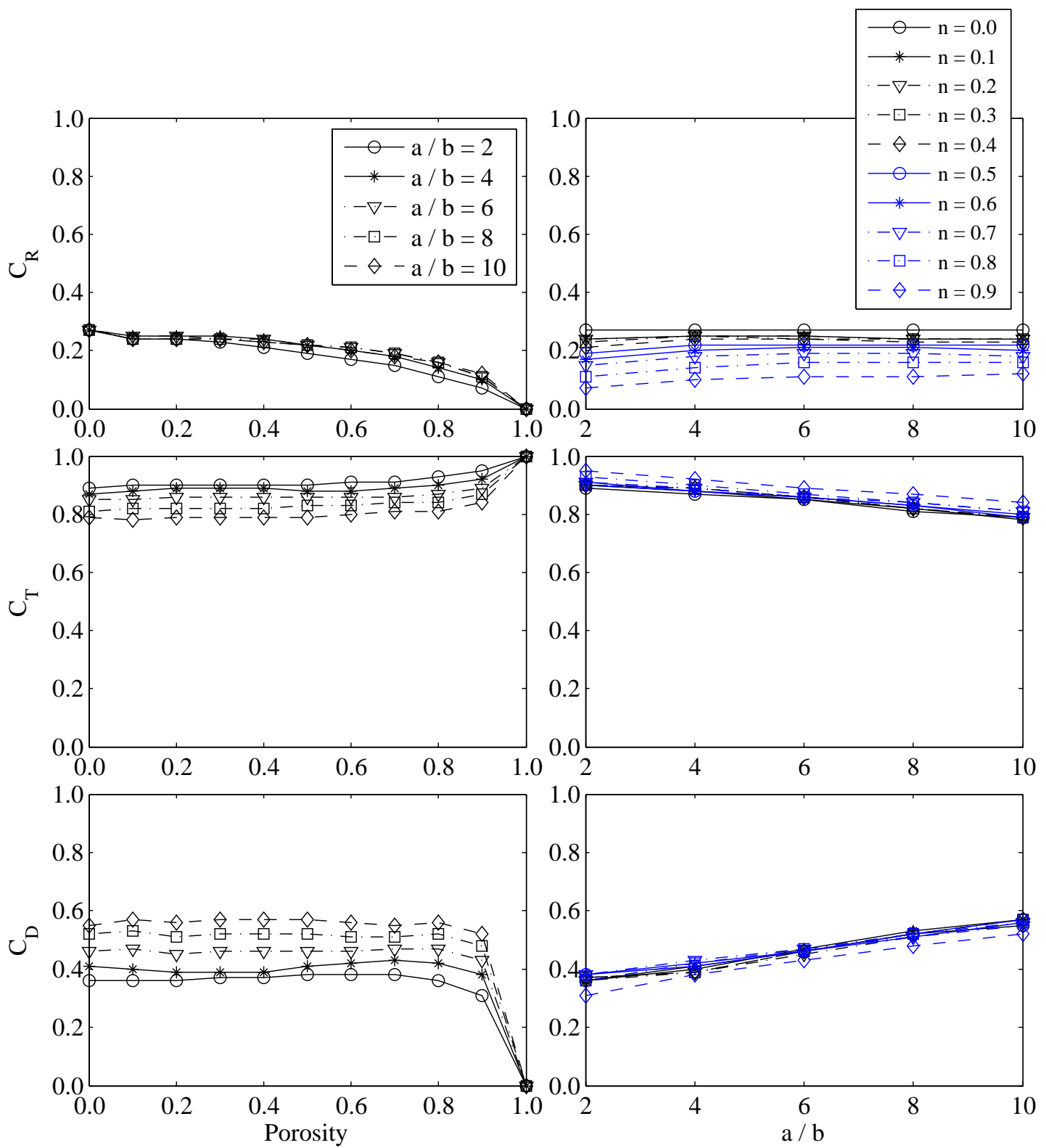

Figure 11. Effects of porosity and aspect ratio $(a / b)$ of the porous obstacle on RTD coefficients. 


\section{CONCLUDING REMARKS}

In this paper, we present the interactions between an isolated solitary wave and a submerged permeable breakwater using both experimental and numerical approaches. The measurement using a PIV system provides the mean free surface elevation and the mean velocity properties in the vicinity of the porous breakwater. Two considerations of porous breakwater in numerical modeling are examined. One is to model a $2 \mathrm{D}$ volume-averaged porous media, and the other is to simulate the real $3 \mathrm{D}$ porous structure composed by uniform spheres as we used in experiments. The comparisons indicate that the 3D LES model can resolve the velocity characteristics in more detail and accurate subject to the tradeoff of requiring more simulation times. The 2D VARANS model can resolve most important phenomena of wave dynamics, and it just needs less simulation times than 3D numerical modeling.

We use 3D numerical results to trace the trajectories of fluid particle around the breakwater to help understand the possible sediment transport for suspended loads. For those particles initially located near the weather edge of the breakwater, most of them starts offshore and moves in the onshore direction, which implies possible scouring at the foot of the coastal structure. For those particles initially located near the lee side of the breakwater, most of them would be driven by the main vortex and finally accumulate in the lee side of obstacle.

The hydraulic performance of the submerged permeable structure between 2D and 3D numerical results is found to be almost the same. Therefore, the effects of porosity and aspect ratio of obstacle on RTD coefficients are investigated by only use of 2D VARANS model. We found that the energy reflection coefficients are dominated by porosity values. More specifically, the energy reflection coefficients decrease with increasing the porosity values. On the other hands, the length of the submerged breakwater has significant effects on the energy transmission and dissipation coefficients. As the length of breakwater increases, the energy transmission coefficients decrease and the energy dissipation coefficients increase.

\section{ACKNOWLEDGMENTS}

This research was financially supported by the National Science Council, Taiwan, under grant NSC 101-2628-E-006-015-MY3. The authors wish to express their gratitude to Prof. Philip L.-F. Liu, Prof. T.-J. Hsu, Prof. A. Torres-Freyermuth, Prof. T.-R. Wu and Dr. K.-C. Hu for their valuable comments on the numerical model. We would also like to acknowledgement Dr. J.-F. Lin for helping the experiments and Mr. C.-L. Yeh for designing 3D numerical modeling.

\section{REFERENCES}

Adrian, R.J. 1991. Particle-imaging techniques for experimental fluid mechanics, Annual Review of Fluid Mechanics, 23, 261-304.

Chorin, A.J. 1968. Numerical solution of the Navier-Stokes equations, Mathematics of Computation, 22 (104), 745-762.

Hirt, C.W., and B.D. Nichols. 1981. Volume of fluid (VOF) method for the dynamics of free boundaries, Journal of Computational Physics, 39, 201-225.

Hsu, H.-C., Y.-Y. Chen, and H.-H. Hwung. 2012. Experimental study of the particle paths in solitary water waves, Philosophical Transactions of the Royal Society A: Mathematical, Physical and Engineering Sciences, 370 (1964), 1629-1637.

Hsu, T.-J., T. Sakakiyama, and P.L.F. Liu. 2002. A numerical model for wave motions and turbulence flows in front of a composite breakwater, Coastal Engineering, 46, 25-50.

Hu, K.-C., S.-C. Hsiao, H.-H. Hwung, and T.-R. Wu. 2012. Three-dimensional numerical modeling of the interaction of dam-break waves and porous media, Advances in Water Resources, 47, 14-30.

Huang, C.-J., H.-H. Chang, and H.-H. Hwung. 2003. Structural permeability effects on the interaction of a solitary wave and a submerged breakwater, Coastal Engineering, 49 (1-2), 1-24.

Lara, J.L., I.J. Losada, M. Maza, and R. Guanche. 2011. Breaking solitary wave evolution over a porous underwater step, Coastal Engineering, 58 (9), 837-850.

Lee, J.J., E. Skjelbreia, and F. Raichlen. 1982. Measurement of velocities in solitary waves, Journal of the Waterway, Port, Coastal and Ocean Division, 108 (2), 200-218.

Lin, P. 2004. A numerical study of solitary wave interaction with rectangular obstacles, Coastal Engineering, 51 (1), 35-51.

Lin, P., and S. Karunarathna. 2007. Numerical study of solitary wave interaction with porous breakwaters, Journal of Waterway, Port, Coastal, and Ocean Engineering, 352-363. 
Lin, P., and P.L.F. Liu. 1998. A numerical study of breaking waves in the surf zone, Journal of Fluid Mechanics, 359, 239-264.

Liu, P.L.F., P. Lin, K.-A. Chang, and T. Sakakiyama. 1999. Numerical modeling of wave interaction with porous structures, Journal of Waterway, Port, Coastal, and Ocean Engineering, 322-330.

Lynett, P.J., P.L.F. Liu, I.J. Losada, and C. Vidal. 2000. Solitary wave interaction with porous breakwaters, Journal of Waterway, Port, Coastal, and Ocean Engineering, 126 (6), 314-322.

Raffel, M., C.E. Willert, and J. Kompenhans. 1998. Particle image velocimetry, Springer.

Synolakis, C.E., and E.N. Bernard. 2006. Tsunami science before and beyond Boxing Day 2004, Philosophical Transactions of the Royal Society A: Mathematical, Physical and Engineering Sciences, 364 (1845), 2231-2265.

Torres-Freyermuth, A., J.L. Lara, and I.J. Losada. 2010. Numerical modelling of short- and long-wave transformation on a barred beach, Coastal Engineering, 57 (3), 317-330.

van Gent, M.R.A. 1995. Wave interaction with permeable coastal structures, Ph.D. Thesis, Delft University of Technology.

$\mathrm{Wu}$, T.-R. 2004. A numerical study of three-dimensional breaking waves and turbulence effects, Ph.D. Thesis, Cornell University.

Wu, Y.-T., S.-C. Hsiao, Z.-C. Huang, and K.-S. Hwang. 2012. Propagation of solitary waves over a bottom-mounted barrier, Coastal Engineering, 62, 31-47. 